OPEN ACCESS

Edited by:

Tom Crick,

Swansea University, United Kingdom

Reviewed by:

Maria Antonietta Impedovo, Aix-Marseille Université, France

Sarina Chugani Molina,

University of San Diego, United States

*Correspondence:

Allison Littlejohn allison.littlejohn@gmail.com

Specialty section:

This article was submitted to

Digital Education,

a section of the journal

Frontiers in Education

Received: 29 April 2019

Accepted: 25 September 2019

Published: 15 October 2019

Citation:

Littlejohn A, Charitonos K and Kaatrakoski H (2019) The Role of Professional Learning in Addressing

Global Challenges: Tensions and

Innovations Associated With AMR

Front. Educ. 4:112

doi: 10.3389/feduc.2019.00112

\section{The Role of Professional Learning in Addressing Global Challenges: Tensions and Innovations Associated With AMR}

\author{
Allison Littlejohn ${ }^{1 *}$, Koula Charitonos ${ }^{2}$ and Heli Kaatrakoski ${ }^{3}$ \\ ${ }^{1}$ School of Education, College of Social Sciences, University of Glasgow, Glasgow, United Kingdom, ${ }^{2}$ Institute of Educational \\ Technology, The Open University, Milton Keynes, United Kingdom, ${ }^{3}$ University of Helsinki Open University, Helsinki, Finland
}

Changing work practice is critical when addressing global challenges. The expansion of work is mediated by a range of tensions inherent in the complex systems within which global challenges exist. This study examines tensions that inhibit the expansion of work practices contextualized within the global health challenge of Antimicrobial Resistance (AMR). The study traces how an AMR surveillance system is being set up in a low-to-middle-income country in Asia (Country A). The research identifies a range of tensions that need to be considered when designing technology-enhanced learning interventions for professionals. This study is significant in moving technology-enhanced learning toward a wholistic approach that takes into account the work environment. This research takes an original standpoint by placing attention on specific work practices, then examining how technology-supported activities can build capacity. This places professionals at the center of a critical approach examining the ways technologies can add value to their professional lives. This work highlights the importance of professionals' "voice" as a lens through which researchers document their reality. The study calls for a fundamental shift in the orientation of technology-enhanced learning interventions, moving attention toward work practice and mapping supporting technologies around this, rather than focusing primarily on the technology and planning learning activity with technology tools.

Keywords: professional learning, technology-enhanced learning, work-integrated learning, AMR, health work

\section{INTRODUCTION: INTERDISCIPLINARY RESEARCH TO ADDRESS GLOBAL CHALLENGES}

There has been a growing interest in challenge-led disciplinary and interdisciplinary research where public, third sector, and private bodies are keen to support research that addresses major, societal challenges. One of the biggest public health challenges of our time is antimicrobial resistance (AMR), defined as the ability of a microorganism (bacteria, viruses, parasites) to stop an antimicrobial (an antibiotic, antiviral or antimalarial) from working against it (World Health Organization, 2019). A consequence of AMR is that standard antibiotic treatments are becoming ineffective and infections are persisting and spreading across populations. Reducing this threat is a public health priority that requires collaborative global health approaches such as the adoption of a Global Action Plan on Antimicrobial Resistance at the World Health Assembly in 2015. To be effective, this type of Global Health Plan has to be followed up by operational progress around key areas where change will have greatest impact. One of these areas is changing professional practice, through targeted professional learning. 
This paper focuses on addressing the global challenge of AntiMicrobial Resistance. The study is part of a major UK Aid initiative (The Fleming Fund) that aims to improve global surveillance of Antimicrobial (AMR), specifically in low- and middle-income countries (LMICs), where the impact from AMR is expected to be particularly acute. The paper attends to the work practice of laboratory professionals working in Public Health laboratories in LMICs, integrating tensions that inhibit changes in practice and questioning whether and how practice change might be supported through technology-enhanced learning (TEL). The study is prompted by the urgency to tackle the complex issue of AMR, alongside the wider discourse around rapidly changing work practices and availability of more sophisticated technology solutions. Although the emphasis of this paper is on the tensions that inhibit appropriate work practices to tackle AMR, the paper makes a significant contribution to research into technology-supported professional learning of laboratory workers. In-depth contextual examination of established work practices of laboratory professionals provides a foundation for developing technological solutions that can add value to these workers' professional lives.

Public health surveillance is the ongoing, systematic collection, analysis, interpretation, and dissemination of data regarding a health-related event for use in public health action to reduce morbidity and mortality and to improve health (World Health Organization, 2019). Public health laboratories have been perceived as at the margins of health work and have been neglected in clinical and animal health sectors, as evidenced by high turnover of staff. Dominant hierarchies and low pay have led to staff feeling undervalued. Although recent developments in drug resistance may be viewed as giving laboratories a prominent place in public heath ecosystems (Morency-Potvin et al., 2017), in LMICs pathology and laboratory medicine services largely are perceived as a neglected part of health systems (Wilson et al., 2018). In our study we view laboratories as a professional setting that should serve as the foundation for a safe and effective health-care delivery and global health security, aligned to the recent positioning of laboratories as important sites for health surveillance. Yet, this expectation places laboratory workers under pressure to expand work practices associated with surveillance.

There is widespread recognition of the need to provide learning opportunities for laboratory professionals, to support them in developing new forms of AMR practice. Yet, the expansion of work is mediated by a range of tensions inherent in the system. These tensions include misalignment of current job roles with the forms of collaboration needed for new work processes; entrenched practices, limiting new ways of working; limited workforce capacity, particularly in specialized areas needed to carry out new forms of work; inadequate infrastructure and quality standards needed for accurate data measurement (Wilson et al., 2018). While it is known that specific tensions inhibit professional learning in laboratory settings, these have not been examined through empirical research. Therefore, the significance of this study is that it provides empirical evidence of tensions that inhibit the expansion of professional practice in AMR surveillance. These tensions provide a platform from which to design blended or technology enhanced solutions to support professionals as they learn.

Technology-Enhanced Learning (TEL) research frequently takes a design perspective, where learning activities are designed around knowledge objectives and the learning is then applied to the workplace. In this work, we take a different standpoint by first placing attention on specific work practices, then examining how technology tools add value to professions by supporting the expansion of practice. To achieve this aim, the research is structured around two research questions:

- What tensions around surveillance practice inhibit the expansion of work practices of lab professionals?

- What tensions need to be taken into account when developing TEL implementations (guidance for TEL design)?

The study was carried out in 2018 in Country A, a small, lowincome country in Asia. Country A was selected as the context of our study because it provided a national context where a National Action Plan on AMR was being approved and introduced by the Government, affording opportunity for the lifecycle of the research to align well with the country developments hence the research findings to shape the development of the AMR surveillance system over time.

This research examines how AMR data are based on and are shaped by established and emerging work practices in the context of surveillance in Country A. The study positions Data associated with AMR as a key object within surveillance activity. These Data include, phenotypic data (growth patterns) or genotypic (presence and expression of genes) characteristics of bacteria categorized according to origin (intrinsic vs. acquired resistance) or type (single, multiple, or cross-resistance) (Davison et al., 2000). Data serve as a focal point for lab activity and for interactions across distributed teams of health professionals working within the public health system.

The paper is structured as follows: First, we set out AMR as a global challenge, reflecting on the ways professional practices need to expand to reduce the effects of AMR. Next we reflect on the relationship between professional work and learning. The research context and method for the fieldwork are provided, followed by discussion of specific examples of professional work. The article ends with a set of conclusions about the future of professional learning for global challenges, examining implications for TEL research.

\section{PROFESSIONAL WORK AND LEARNING IN RELATION TO AMR SURVEILLANCE}

\section{The AMR Surveillance System}

Globally AMR surveillance provides early warning about the spread of new resistant strains of bacteria, illustrating geographic variations in the incidence prevalence of resistant pathogens and helping to identify long-term trends. A UK government report (O’Neil, 2014) identified poor AMR surveillance as a major inhibitor to slowing down bacterial resistance, since AMR data can help inform at a local level the best treatment and care for individual patients. At the regional level, surveillance data 
informs intervention priorities and gaps in service delivery. At a national level, data highlights those populations most at risk, thereby guiding planning and resource allocation and informing health policies and responses to patterns and trends.

The foundation of AMR surveillance systems are the local surveillance sites, where data are generated, collated and reported in ways that provide national and international overviews of AMR. AMR Data are important for clinicians to help them prescribe for each patient the "right antibiotic at the right dosage for the right amount of time" (Hall, 2018). This process is especially challenging in LMICs, where the environment is complex and challenging because of limited infrastructure and where AMR surveillance work is fragmented. Yet their work not only underpins national health-care systems, but contributes to global surveillance systems. Efforts to improve the operation of local laboratories in LMICs have been local, fragmented, and mostly unsustainable (Sayed et al., 2018). Yet, these local surveillance facilities are critical to provide good quality AMR surveillance data to inform regional, national and international health systems on how to respond to AMR trends.

At the local level, a patient visits a doctor with an infection. The doctor takes a sample (e.g., urine, blood) and sends it to the hospital lab to be tested. Hospital laboratories routinely perform clinical tests on patient samples to provide clinicians with data that is used to diagnose and treat the patient. These data are gathered from a number of hospital labs and are collated for use as regional or national surveillance data. Local AMR surveillance is carried out in a variety of ways, summarized by Figure 1:

Local surveillance practice comprises five broad task areas, all of which are critical for good quality data:

- Collecting/Receiving specimens and logging
specimen information;
- Performing tests (e.g., preparation of media, growing culture);
- Monitoring and documenting data;
- Interpreting data as test results;
- Reporting and communicating results/data beyond the
laboratory (local/national/global).

Responsibility for surveillance is distributed across various professionals, including clinicians or nurses who gather samples, lab technicians who perform tests, senior lab professionals to interpret data and report data to facility management or regional officers. Good inter-working with a local, distributed team is critical for high quality Data. In this way, Data can be viewed as an important object around which professionals work during AMR surveillance. As Data is distributed up from local to regional systems, professionals become more loosely bound by national surveillance system activity and work and need to work as expanded team (e.g., in the ward, in an office, in a laboratory, in another facility). However, the worldwide AMR surveillance system is dependent on good quality local surveillance data, therefore, the focus in this study is on a team in a specific facility where surveillance tasks are shared amongst people with different job roles. Local surveillance work needs to be expanded and continually updated, as new AMR surveillance practices and techniques are developed. This means that professionals in public health facilities need continually to expand their practice and make sure they have up-to-date knowledge about surveillance techniques and data interpretation. The next section gives a background to the embryonic AMR surveillance system in Country A.

\section{AMR Surveillance in Country A}

Country A is an Asian country that is among the least developed countries in the DAC List of Aid recipients ${ }^{1}$. It was selected as the context of our study because at the point that the research team visited the country in July 2018 an AMR surveillance system had been only recently introduced. During our visit in July 2018, country officials were further in the process of negotiating their grant agreement with the Fleming Fund, so our research activities would coincide with the country's participation in the global project. As a result, Country A provided an interesting case to examine, especially since we sought to examine what forms of expanded professional practices emerge as a result of introducing an AMR Surveillance system and what tensions can be observed that inhibit professionals from developing new practices.

At the time of our field visit, the country had made good progress on the One Health Approach and developed a One Health Strategic Plan (2017-2021), though it had not yet been fully implemented. It had also endorsed setting up the permanent One Health Secretariat. Responding to the WHO global action plan guidelines, in 2017 Country A had launched a National Action Plan (NAP) for AMR (2018-2023), covering human and animal health. To take forward the NAP, the Government established a dedicated AMR programme within the Ministry of Health and the Livestock Sector. This was overseen by a crosssectoral AMR Committee with a dedicated AMR Programme Officer who was appointed to oversee national AMR activity and the introduction of the role of the AMR Focal Person in each sector. The country has developed National Antibiotics Guideline for use in both humans and animals, whilst over-the counter sale of antibiotics without prescription is banned. Furthermore, use of critical antibiotics is restricted in animals and the use of antibiotics in animal feed is banned. However, despite the development of good regulatory policies and guidelines on antibiotics use in humans and animals, these are not fully implemented due to lack of resources.

Laboratory personnel capacity varied substantially across different locations in Country A, with rural settings having limited human resource compared to urban areas. AMR expertise was concentrated in the AMR reference labs and central labs in the capital city; few labs had international accreditation; as in most countries, AMR surveillance within the human health sector was better developed than in the animal health sector; and the number of qualified microbiologists in the country was low. Day-to-day operation of the laboratories was affected by a number of systemic issues, including infrastructure (i.e., power supply, equipment, lack of capacity to use the equipment), quality (i.e., quality control, quality assurance), procurement processes and access to high quality reagents. An AMR surveillance network was being set up through: a

${ }^{1}$ OECD DAC ODA list http://www.oecd.org/dac/financing-sustainabledevelopment/development-finance-standards/daclist.htm 


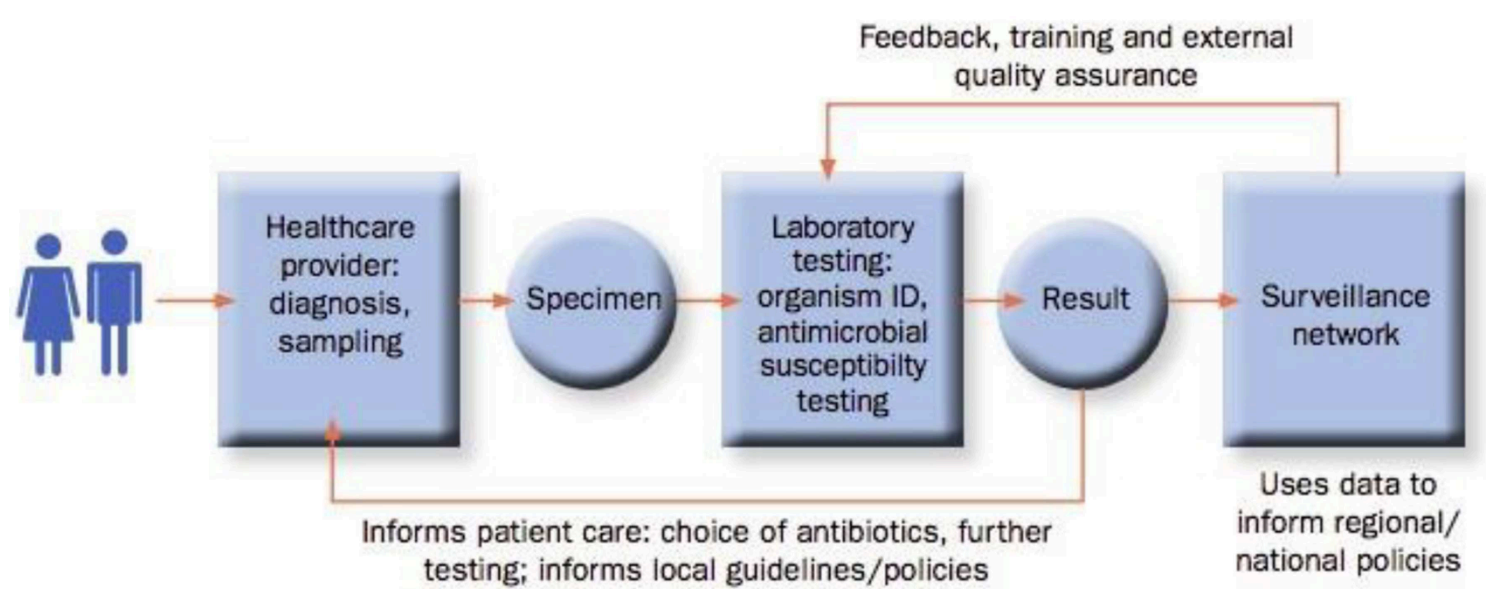

FIGURE 1 | Local AMR surveillance process (source: https://amr.lshtm.ac.uk/wp-content/uploads/sites/12/2016/11/AMR-Surveillance-Protocol.pdf).

human heath National Reference Laboratory and four sentinel sites; an animal health AMR reference laboratory, four Regional Laboratories and a National Food Testing Laboratory and a National Feed Laboratory. In human health, laboratory capacity for culture and sensitivity of bacteria isolates was available and there was additional AMR capacity at two regional level hospital laboratories and one district level hospital. There were no microbiology laboratories at the other district hospitals within Country A. Similarly, animal health AMR susceptibility testing was carried out in the reference lab. All other animal health sites had the capacity to culture and isolate bacteria, but antibiotic susceptibility testing was not carried out due to resourcing issues.

There was general recognition of the need to develop and expand current forms of professional work on AMR surveillance through professional learning. However, consideration of how this might be achieved tended to focus on conventional forms of learning through a curriculum, rather than by considering the relationship between professional work and learning.

\section{Professional Work and Learning}

Consideration of professional learning tends to focus on formal training, where large numbers of people are supported in reaching a specific level of competency in a curriculum with preprescribed learning objectives (Littlejohn and Margaryan, 2014). However, professional learning is influenced by intrapersonal factors such as previous knowledge, skills and attitudes, and is also shaped by the work environment, job roles, tasks and culture (Littlejohn et al., 2016a). As people deal with constant changes in the work environment, they need to learn new forms of knowledge and practice on an ongoing basis to solve emerging problems (Hager, 2004; Hadwin et al., 2011; Illeris, 2011). Understanding learning for work, therefore, requires knowledge from diverse areas including sociology, examining the social relations, normative codes, and organizational structures that inform the practices, experience, and identities of professionals; educational psychology, investigating the cognitive, affective and behavioral factors influencing learning at work; education, focusing on pedagogy and learner agency needed to develop new skills and knowledge for work; and computer science, examining computational thinking and the assumptions that underpin programming decisions in relation to TEL. Currently these areas are viewed as distinct fields and, there is insufficient attention to the analysis of work practices and the workplace as key variables of learning.

Work practices tend to be passed down through generations. They become systemic and associated with "being" a practitioner. When newcomers come into a workplace they try to fit in and become "enculturated" into systemic practices and ways of doing things associated with expertise (Williams, 2014). Known "ways of doing" are engendered in generations of practice and, whether good or bad, tend to endure as a template of "how it is to be" a practitioner. It can be very difficult to change these practices, even when they become so outdated that they no longer work well. Any challenge to entrenched practices not only has to be sound and well-argued, but also has to be aligned with or change the work culture. Entrenched poor practice is a major contributor to transforming work. Although the organization of work sets the conditions for learning, it is the interaction of the learner with the environment that determines learning (Tynjälä, 2008). Professionals, therefore, have to be active agents, self-regulating their own learning, particularly where they are working at the boundaries of knowledge and cannot rely on courses to expand their knowledge (Littlejohn et al., 2016b). This dialectic relationship between work and learning is important, therefore as we argue in this paper it is critical to consider work practices and learning processes together at the same time within the context of the work environment, rather than treating these factors as separate.

Consideration of the impact of the workplace on learning is particularly relevant, since there is growing recognition that there are few situations where professional learning takes place formally, without also informal learning through routine work tasks (Colley et al., 2002). Eraut (2011) emphasizes the importance of informal (or "non-formal") learning at work. As professionals learn through routine work tasks, the workplace acts as a site for learning (Boud and Garrick, 1999). Learning 
AMR Surveillance Networks

Reference Labs

Sentinel Sites / Surveillance Labs

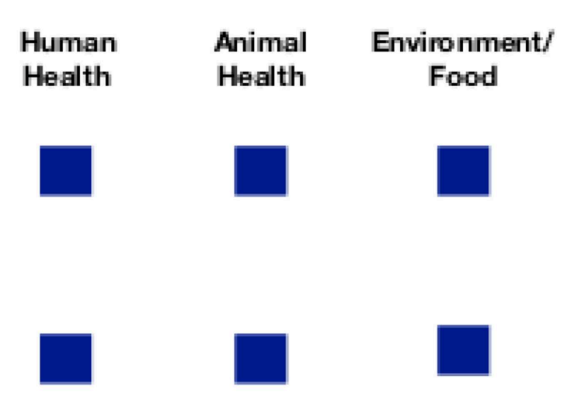

Country A:

FIGURE 2 | Sites selected for fieldwork in Country A. and development opportunities, therefore, are situated within the workplace and co-exist with expert practice (Boshuizen, 2004). The workplace context and culture influences and shapes learning, as professionals expand and develop their practice (Fuller and Unwin, 2003; Unwin and Fuller, 2004).

Similarly, the workplace technologies and how these tools support professional learning have to be considered within the context of work and work practices (Littlejohn and Margaryan, 2014). For example, technology tools can be used to augment informal learning at work. A common way of learning at work is through feedback on day-to-day work activities from colleagues with more expertise. Technology tools can be used to harvest and exploit the feedback people receive, gathering feedback and disseminating it for reuse (Boshuizen, 2004). A simple example is recording a video with verbal feedback and uploading this to a social media site (e.g., YouTube). In this way, social media digital networks provide dynamic environments that connect work and learning through collaboration around "objects of inquiry" or shared work objectives (Paavola et al., 2004; Paavola and Hakkarainen, 2005). Thus, digital technologies offer opportunities for the individual to work and learn with the collective (Engeström, 2007). Yet the use of networked technologies to capture and exploit expert feedback and to connect the individual with the collective knowledge remains under-developed. This issue emphasizes the need to consider technology use and development within the context of the workplace. Fieldwork is necessary to examine the tensions that inhibit the expansion of work practices and to identify which of these need to be taken into account when developing implementations with technological tools.

The next section provides a description of the research context and fieldwork and details the methodological approach used to generate data to answer the research questions.

\section{METHOD AND CONTEXT OF STUDY}

Data collection took place in July 2018 in Country A (see section AMR Surveillance in Country A). Prior to data collection, the researchers followed the ethical approval procedures of the university, having ethical approval of the method, instruments and process for data gathering, analysis, and storage. All participants in the study had a full explanation of the purpose of the study along with their rights to choose whether to participate and to withdraw any data pertaining to them at any time. Data was gathered through field observations and semi-structured interviews, as outlined in the next sections.

\section{Site Selection and Fieldwork}

Desk-based research was carried out to select sites to be visited. This work involved scoping key reports, such as a National Action Plan for AMR prepared by Country A's Health Ministry, in partnership with the World Health Organization (WHO). Six laboratories across human health, animal health and agriculture sectors were selected for visits over a 10 day period in July 2018. These laboratories were part of the Country A's surveillance network. Sites included two reference labs, two central facilities located in the capital city and two district facilities, as illustrated in Figure 2. By selecting these sites we had good representation of different facilities across both urban and rural areas. These site visits were endorsed by the AMR Committee in Country A.

Two sources of qualitative data were gathered: first, fieldnotes from site observation in each of these six laboratories, mainly collected through "guided tours." These tours were organized for the research team by laboratory staff in each site and included meetings with heads of units and wider laboratory teams. Our fieldnotes comprise of notes and photos. Second, interviews with 25 professionals across these sites ( 22 individual interviews and one group interview with 3 professionals). This sample included 19 men and 6 women, which is representative of the gender balance across the sites. Interviews were conducted in English. Each visit began with an introductory meeting with members of staff in the facility to allow the research team to introduce themselves and explain the purpose of the visit. This initial meeting was followed by individual interviews with professionals working in specific job roles (see Table 1). Professionals were purposefully selected to allow representative 
TABLE 1 | Interviews conducted in Country A.

\begin{tabular}{lc}
\hline Professional roles & Country \\
\hline Laboratory professionals & 9 \\
Senior laboratory professionals & 10 \\
Clinical services professionals & 1 \\
Senior management staff in clinical services & 1 \\
Policymaker & 3 \\
AMR community/expert & 1 \\
\hline Total & 25 \\
\hline
\end{tabular}

sampling of specific job roles across the facility with responsibility for AMR surveillance.

Interviews lasted $30-60 \mathrm{~min}$. Each interview was guided by a semi-structured instrument previously used in studies of selfregulated, professional learning (Littlejohn et al., 2016a). Each interview was audio-recorded and transcribed verbatim. Table 1 illustrates the professional roles of those interviewed.

\section{Data Analysis}

Thematic analysis for the interview data was performed using the thematic categorization method described by Tuomi and Sarajärvi (2009). The reason this method was selected was because it places primacy on the thematic contents of the data, supporting detailed analysis of large-scale qualitative datasets. The interviews were loaded into the analysis software application, NVivo 11. Data were analyzed within the framework of cultural-historical activity theory (Engeström, 2015) and involved assembling a range of challenges associated with professionals' work that were articulated during the interviews. The key concepts driving the analysis were "subject of activity," "tensions within activity," and "actions within activity."

The data analysis was guided by a number of key questions: "What are key activities organizations/laboratories do?"; "What do individuals say they do"; "What does the practice linked to surveillance look like?"; "What are the perceived challenges in their work?" and "How do individuals say they learn and develop new skills and knowledge in their work?". Our primary aim was to synthesize knowledge and develop an in-depth understanding of the characteristics of the laboratory setting, to identify the various roles within laboratory settings and trace their working relationships with other professionals in public-health facilities. We placed particular attention to current forms of work practice in AMR surveillance and forms of professional learning that are used to build capacity in this context, as well as the different ways in which technology is used to support professional practice in this context.

Following this, our analytical approach focused on performing a thorough mapping of knowledge and skills gaps per professional role (see Charitonos et al., 2018). Analysis of these data enabled identification of tensions between elements of the AMR surveillance activity system at the country level, that were coded drawing on Engeström's method (Engeström, 2015). The latter is of particular importance as it illustrates the work context and identifies issues with ways of working amongst professionals. Drawing on this analysis, the paper highlights problems of surveillance practice in public health settings. Following on from this, we consider forms of learning activities to support lab professionals in learning relevant knowledge and practice and consider how technologies might be used to support this learning in the workplace.

Systemic issues affecting ways of working in AMR surveillance systems and shaping work practice are discussed further in the next section.

\section{RESULTS AND DISCUSSION ABOUT PROBLEMS OF PRACTICE}

The fieldwork aimed to address the research question, "What tensions around surveillance practice inhibit the expansion of work practices of lab professionals?" Building on the rigorous data collection and the systematic method of analysis described in section Results and Discussion About Problems of Practice, three significant tensions were identified. The first of these was the tension between a perceived need for professionals to take time out of work to learn about new ways of working and the limited human resource available with the capability to undertake surveillance activity. A second tension was implementing new ways of working within existing work settings, with limited opportunity to re-configure the work environment to support new practice. The third tension focused around one the one hand inter-relationships of professionals as new work practices evolve and, on the other hand, entrenched professional norms such as rigid professional hierarchies and long-established work processes. In conventional microbiology practice, laboratory professionals work in a disconnected way. However, modern AMR surveillance practice is based around the creation and dissemination of data across local sites and national/international sectors. These findings are illustrated using narrative accounts from eight professionals $(n=8)$ interviewed in one particular human health facility located in an urban area. These 8 interviews are representative of the study's wider findings (see Charitonos et al., 2018) and are selected as their high number and diversity in roles allow an in-depth examination of work practices in a particular human health facility in Country A. It is important to note the range of roles of the interviewees, across clinical and lab services and across ranks, as shown in Table 2.

In local Facilities we observed limited understanding of how to support professional learning, how to adapt work environments and how to reconfigure work relationships. Our understanding of these tensions is blurred and not yet adequately negotiated, developed or embedded. One way to resolve these tensions is to position "Data" as a key object within the surveillance activity that is both based on and shaped by professional practice and work configurations. Through these case examples we unpack how Data exists in various forms, modalities and understandings among professionals. We further illustrate how these Data are constructed by professionals through the evolution of action over time. We highlight that in order to move from current ways of working to new work practices around the creation and 
TABLE 2 | Job profile of participants.

Senior management-clinical services

Clinical services professional/AMR clinical-lab

Senior laboratory professionals

Laboratory professionals

dissemination of Data, learning has to be in parallel with the reconfiguration of both work relationships and environment.

\section{Limited Human Resource}

Human capacity to address AMR is a major issue in Country A. There was a general consensus that professionals in different roles lack knowledge about AMR, as in the following quote which illustrates a typical response:

\begin{abstract}
"Difficulties? Well, one thing I found out when this terrible situation arose [patient with high levels of resistance to antibiotics] is that we [facility] didn't have many people who knew much about what to do. Probably that is the main problem we are having, because when I asked the infection control team, OK, what are you going to do? So, they didn't know so readily. They might have to look up. So basically, we don't have people who are trained and have the knowledge of what should be done in case something like this comes up. So that was one of the main issues... I also don't have much idea..." [P1, senior management role in Clinical Services]
\end{abstract}

Few professionals were appropriately trained, limiting their ability to expand their practice to include new ways of working. One Laboratory Scientist explained: "we are not having enough people to work around the clock" [P1, senior management Clinical Services], hence "the top difficulty... is when people go out of station" [P3, Laboratory Scientist]

There was a high volume of routine work that the laboratory staff routinely carried out, in which they performed the same laboratory tests and tasks year after year. They perceived a need to focus on these routine tasks and believed they had limited opportunity to expand their practice. This had an impact on workers' capacity and motivation:

\footnotetext{
“I haven't seen much opportunity [to upskill]... Most of my four years, I have spent doing media and a little bacteriology... And I think it's all daily basis work, the 9:00 am to 3:00 pm work, and that's it... Primarily, we don't get much opportunity and I don't have much time to take interest in the microbiology because we, like, can't take time to focus more than what I primarily do at work. Because we are occupied...." [P3, Laboratory professional]
}

Another problem was that workers were not trained in critical tasks, such as preparing reports (for example antimicrobial susceptibility report or antibiogram). This not only limited their experience and understanding of the AMR surveillance system and how they should evolve their practice, but it also reduced their ability to perform routine tasks associated with AMR surveillance. For example, when clinicians were sent AMR susceptibility reports, few knew how to interpret them and reports were not written in a way that would help clinicians make an informed decision about what antibiotic to prescribe. Yet, there was little attempt to support lab professionals and clinicians in working together to improve inter-work and communication. Lab professionals tended to work in an insular way -which is how they had been used to working-focusing on the work in the lab. This could be because they had limited opportunity to meet and interact with the professionals who were "upstream" (e.g., Clinicians) or "downstream" (e.g., Health workers who collect bacteria samples) within the AMT Data pipeline.

Senior staff were most likely to be offered opportunity to travel for professional development. Responding to the question "How do you go about developing new skills and knowledge on AMR?", some of the more Senior Lab professionals described how they would leave the workplace for training, either as a placement in a partner laboratory or to study for a qualification abroad (such as an $\mathrm{MA}$ or $\mathrm{PhD}$ ), supported by the state. One professional in clinical services explained why one senior colleague was supported to go to "England for a 3-6 months course, because we [senior management] thought, if she [professional] learns and comes back, she could help out" [P1, senior management Clinical Services] By spending time abroad at an advanced Facility, this senior colleague was able to develop deep, more specialist knowledge that she used to support the expansion of work practice and redevelopment of work environments across Country A.

However, in a small country with limited human resource, taking professionals out of their job in order to develop them sets up a paradox: on the one hand people need to have access to opportunities for re-skilling or upskilling in relation to AMR, but these opportunities are limited where there are restriction on the size of the talent pool available.

The provision of continuous training is important. However, access to professional development was restricted because of the limited availability of human resource to replace people who tended to be out of the workplace while engaging in training. The issue of professionals taking time out of work to update their knowledge was exacerbated by the increasing specialization of job roles. Knowledge of specialist practice made it more difficult for roles to be filled by another lab worker when someone took time out for training. One positive way to support professional learning was through a rota system that allowed everyone to have on-the-job upskilling and gain experience of a range of tasks. This means that when someone is absent, someone else can carry out the work. For some people traveling or attending to training during work hours can never be an option, due to caring or other responsibilities, disadvantaging professionals. Opportunities to learn while at work appeared crucial:

\footnotetext{
"And it's not always about opportunity. It's about several small quantities, small CMEs [professional learning], like, having a small workshop- one to two hours' workshop- where people can sit and share experience...We did it a few years back. But some of it stopped because we couldn't prioritise those workshops over our routine works. So 9:00 to 3:00, you work. After that, you are tired. So either you want to go home because we have [INAUDIBLE] too. We have other works to do." [P3, Laboratory Professional]
} 
Some of the more junior professionals explained they never had opportunity to travel elsewhere for training or professional development:

\begin{abstract}
"So, there is always the conflict when it comes to opportunities. So, OK, let's say someone went for a training, right? It's OK he went for a training... the person cannot [shouldn't] go because he or she is a favourite of someone, favourite of the 'in- charge of things', and not also because they have higher qualifications... We are technicians. We are limited. I understand that we are... So especially when it comes to opportunities, OK, other people, they get disheartened because... the opportunities which are meant for them, which they could have also utilized has not being given to them. And I'm not saying there are a lot of opportunities. But those deserving opportunity have been missed out" [P3, Laboratory Professional]
\end{abstract}

Limitations around who was able to visit other facilities for training limited peoples' opportunity to interact with and build relationships with colleagues in other sites; "They haven't gone outside and looked at what other people are doing, what other hospitals are doing, what other labs are doing. We don't have any idea..." (group interview).

More often human resource development for junior staff was through 'in-service professional development' termed Continuous Medical Education (CME). CME is not freely accessible for lab professionals and Lab managers have to apply for and secure funds for CME, which can be perceived as a time-consuming and bureaucratic process with limited chances of success. One Senior Lab Professional (P 4) explained:

\footnotetext{
"We try to give them [technical staff] the CME, as well. We try to organise some teams... Since we are not autonomous we have to go through the hospital administration... They have to look over not only that, but they have to look all over the hospital (...) We have difficulty in securing the funds now."
}

Managers sometimes introduced informal forms of professional development, such as mentoring. In the facility junior members of staff should have been be mentored by senior staff. However, mentoring was not implemented and embedded in a systematic way, due to the limited human resource. Senior staff felt that they had to prioritize immediate, day-to-day issues over mentoring junior colleagues: "Those we have [in bacteriology], we are not able to every time supervise or be on them, whether they have (done tests) correctly, because sometimes we're not around also. Like today, we have to be running down for many other stuff" (group interview).

Laboratory managers tried to alleviate this tension-making sure sufficient human resource was available to carry out work tasks while, at the same time, providing professional development opportunities-by hosting external experts who would train staff. These experts often traveled traveled from India or Western countries to spend time on-site, providing training for junior staff. However, this training was not always perceived by inhouse staff as relevant to their context of work. As one professional remarked: "this is the problem with them [external experts]... when they come here, some textbooks when they come here, they don't understand our settings. So it's- hands-on it's a little bit difficult to learn from those experts" [group interview]. As one lab scientist explained:

“... I see no use in getting a training for something that the lab can't utilise me for, because I have seen some of my colleagues go out for that particular training. And when they come back, it's just normal routine work. There is nothing new to add to the services" [P3, Laboratory Scientist]

In summary, there are a number of ways professional learning can be implemented, but these approaches have to take into consideration the availability and work commitments of professionals and the environment in which learning takes place.

\section{Restricted Opportunity to Re-configure the Work Environment}

We identified a tension in how existing workplace structures, roles, and rules can hinder or enable emerging surveillance practice to develop and flourish. Surveillance practice requires the local workplace to re-configure their structures and consider roles and processes that may support surveillance. Professional practice is ultimately structured by the workplace. For successful adoption of new practice, and for learning to be effective, people have to be able to reflect on and adapt their workplace as they learn new knowledge and skills. However, there was limited opportunity for re-configuration of the work environment.

This problem is illustrated by considering how, as AMR surveillance work practices evolve, Data has to be persistently available and able to flow within the system. However, one Clinical services professional explained:

\footnotetext{
"We have kept data for, like, last one and a half years. But we have not, you know, used it in terms of analysis or interpretation of the data, because of many reasons. One is because we had everything done manually. So, whenever the person is, like, travelling or on leave, then the data is missing for those periods of time" [P8, Clinical Services professional]
}

This interviewee described how data analysis routinely is carried out "manually" rather than digitally. He explains that the reason that practice around data analysis has not evolved is because the specialized equipment needed to perform tests and analyse data digitally is limited. The scarcity of appropriate equipment means there is also limited knowledge about microbiology techniques. This limitation in knowledge and practice means laboratory professionals cannot identify organisms at the subspecies level, confirming small serologic tests or running specialized tests on equipment, such as a "PCR" machine. Although the PCR was available, it was "under utilized... left idle" [group interview], because of maintenance (rather than training) issues. This mean that data shared at the national and global levels were not detailed for in ways that support effective AMR surveillance.

A key priority for the microbiology Senior Lab Professional was to establish a Quality Assurance system to support the generation of good quality data, underpinned by a review of Standard Operating Procedures (SOP). SOP is a key tool 
for professionals in laboratories that supports consistency and standardization of procedures; "whether I perform a test, whether you perform a test, if we have a standard operating procedure, it doesn't matter, for the uniformity, to standardize this" [P4, Senior Lab professional]. Any change in professional practice requires the SOP to be updated. However, the Facility Administration had released resources to enable the SPT to be revised. This problem was partly due to the fact that, when professionals work in new ways, not everyone in the system has a coherent view of "what Quality Activities (...) that we should have. The division or Ministry, also, they don't have a defined structure of what these quality activities in microbiology... Is it right, the right way that we are doing it?... We are doing pretty much just (...) according to our guideline. That guideline, only. I don't know what other guideline says, you know... There's no reference point for what quality should be, according to the government" [group interview].

This lack of coherence affected other processes within the system. A major issue procurement, where legislation conventionally required staff to order equipment from the supplier with the lowest costs. This legislation caused problems with the purchase of media and reagents, because inexpensive reagents were not sufficiently sensitive to carry out tests at a sensitivity level that could detect AMR. One lab professional explained how they had tried to explain this problem to the procurement administrators, with limited effect: "Still we are fighting for the procurement things. We have convinced the bureaucrats [INAUDIBLE]. We have been trying to develop some proposals ahead to what we can do" [group interview].

One instance when the work environment was successfully reconfigured was when the Facility reviewed its structure, aiming to reduce the incidence of antibiotic prescription and to monitor the prescription of high-generation antibiotics. A new AMR specific role was introduced, to monitor and oversee this work. This professional acted as a liaison between the clinicians and the laboratory supervisor, and participated within local, hospital committees as well as the national AMR committee. The creation of this new role led to several important changes in work practices. For example, in the past clinicians had autonomy over which antibiotic they prescribed for patients. Some high-level antibiotics included "drugs of last resort." These are antibiotics that should only be prescribed in extreme cases, to ensure their effectiveness is not compromised by AMR. This practice was changed such that all prescriptions of antibiotics "of last resort" required approval by the stewardship physician. In the following extract this person describes the process of collecting data and working alongside a pharmacist and clinicians in the process:

\footnotetext{
"what we currently do is the pharmacist goes to all the inpatient departments- that is the wards and the units, like the intensive care unit- and collects the data of those patients who are on any kind of anti-microbial. She does it all manually, because we do not have any IT support where, you know, get all the data directly. So, she collects it every Monday and Tuesday manually. And then she maintains a register where she writes what kind of antibiotics has been prescribed. What was the patient getting it? What is the disease? And what was the duration? And then after,
}

once she finished collecting the data, then I go to these individual wards and see these patients who are put on these antibiotics... I look at many things. Like I look whether this patient really needs this antibiotic, yes or no, first of all. If I think no, then I go and discuss with the prescribing physician. What was his reason for prescribing this antibiotic? And we discuss... And if we both come to a conclusion, OK, we can stop this antibiotic, then we stop. Actually, stewardship is all about that" [P8, Clinical Services professional].

By introducing a new role with authority to change practice, AMR was controlled more effectively. Another advantage of this new role was that it provided specialist knowledge in the wards, allowing transfer of knowledge to nurses and clinicians. This inter-professional work generated more trusted relationships across diverse professional groups. The professionals we interviewed acknowledged that the re-configuration of work through generation of other specialist posts would support the transformation of AMR surveillance practice.

Re-organization of work through the creation of new roles and inter-professional working proved an important way for the Facility to implement new ways of working, support professionals in understanding how their role fits within the AMR surveillance system and how their work relates to the work of others. These inter-relationships are critical for the transformation of work.

\section{Depending Inter-relationships While Implementing New Ways of Working}

Shifting laboratory practice toward new, emerging AMR surveillance practices requires professionals to relate to one another in different ways. These relationship often were conceptualized around the development and conveyancing of Specimens and/ or Data. A number of professionals in the system while described different points in the surveillance process when they would hand over Data to other professionals. For example, specimen samples were handed over from a collection station to the testing site in the laboratory, described by a Lab professional as follows:

Interviewer: Can you describe a task that you do in your day-to-day job? What does it Involve?

Professional 2: So, my job is to collect the sample only [not in the lab]

Interviewer: So, you collect the sample. And then when you come here [lab], do you do the processing as well?

Professional 2: Processing, no. The other people will do.

interviewer: OK, sorry.

Professional 2: As the sample (involves) yeah. So, after finishing the sample collection, then I'll help in data punching (but not the tests) [P2, Lab professional]

This technician's main work task involved sample collection and "data punching" "to enter all the patient details and everythingage, sex, everything in the log" [P2]. As specimen information was logged and processed, various data points were generated and represented as numeric data. These data were sent from one professional located at the laboratory test site to another professional in a different section of the laboratory to be 
documented in a ledger or on a computer system, where the numeric data were aggregated. Finally, these data were translated into a report that was sent to people outside the laboratory (clinicians, nurses, or patients).

This passing on of data requires good relationships and interworking with key people. Nurses, for example, play an important role in AMR surveillance, since they are usually the first to receive the lab reports. One professional described how she recognized the need for nurses to learn about AMR and how this could be encouraged through inter-working:

I'm giving a lot of push to the nurses... Maybe in other countries the lab persons come to the ward and talk to physicians or the pharmacist does that. But in [Country A] we do not have that kind of system. It's the nurses who get all those reports. And they are the ones who have to really be educated what kind of reports, what does it mean, so that they can, you know, tell the physician. [P8, Clinical Services professional]

For the AMR surveillance system to operate effectively, professionals at various points in the system have to understand how their work contributes to the wider surveillance process. Yet most were unaware of how the samples or data they generated were used in the preceding or later points in the system. This problem is evidenced in the interview lab technician interview where, in response to the question "What would you say is good practice in AMR?" he replied:

Professional 2: ... we have been working. But we didn't realise that an AMR is that important. So, we have been punching the data. But we have no idea that this data could be very useful. Interviewer: OK. Do you know where this data goes?

Professional 2: No. We just put in.

Interviewer: So, who sees the data?

Professional 2: (...) he [lab supervisor] does all that.

Interviewer: Mm-hm. And then do you know what does he do with that?

Professional 2: No. But recently, he talked with his (contacts). So, there's going to be an AMR something (...) - research is going on. So-

Interviewer: $\mathrm{OK}$.

Professional 2: But the data we have been punching since long time.

Interviewer: Yeah, yeah, yeah, of course.

[P2, Lab Professional]

Similar issues were observed in other parts in the surveillance process, particularly at points where data results were reported or communicated. For example, where reports were passed from the lab to a clinician or a nurse to support clinical treatment, as illustrated by the following quote:

Professional 8: So, the lab sends the results, like the blood culture, the urine culture... and I make sure that the physicians have seen those reports. What actually used to happen was most of the time these lab reports come back, but most of the time the physicians haven't seen it and yes, so they do not prescribe an antibiotic which is in line with the lab reports. So, we're trying to make sure that they see it and they treat the patient in line with the reports... The most important reason they say is they cannot trust our lab reports.

Interviewer: OK. I was going to ask you.

Professional 8: Yes.

Interviewer: From your experience, is this the reason?

Professional 8: Yeah, so that is one of the most common answer I used to get.

Interviewer: How do they justify that? I mean, what do they say about not trusting it [report]?

Professional 8: So they say we're just a bit of- I mean, truth is there. Because they say most of the time we have done our education from a university where most of the labs, especially the cultures, were done by a microbiologist, a clinical microbiologist. But here, we have more of technicians doing it or a laboratory officer doing it. So, they [clinicians] really doubt what kind of, you know, quality are they producing. Can it be really trusted? [P8, Clinical Services professional]

The professionals we interviewed reported a number of similar critical issues. First, clinicians often did not use lab-diagnostic data to inform the treatment they offered; second, there was a persistent lack of trust between different sets of professionals; third, there were no regular updates regarding the impact of lab-diagnostics have on the effectiveness of patient treatment. These issues signal that capacity building has to extend beyond the development of individual knowledge and skills to include whole system of activity in learning. It is essential to develop forms of learning that support colleagues in understanding their position within the surveillance system and how they inter-relate to others.

\section{HOW KEY TENSIONS MIGHT BE REDUCED THROUGH TEL IMPLEMENTATION}

In this study we re-positioned microbiology laboratories within a public health system as a knowledge-intense environment, highlighting the critical work of lab professionals. These descriptions of AMR surveillance practice offer a lens through which we record and view the lived reality of professionals working in laboratory services. By closely attending to the voices of participants and their experiences, we offer a critical approach to the ways technologies and learning approaches can add value to their professional lives. Therefore, although this study is contextualized within the health sector-specifically AMR surveillance-these findings will likely have applicability across a range of professional learning settings. Particularly in contexts where work continually is changing and where the application of new knowledge requires a simultaneous change in the work environment. In general, professionals' engagement in surveillance activity in public health systems is likely to persist as a worthwhile focus for research in educational and professional learning.

Our research identified three major tensions that inhibit the implementation of AMR surveillance systems. Considering how these tensions might be taken into account when developing TEL implementations, provides guidance for future TEL design. 
The first tension relates to the increasing pressures being placed on professionals in public health facilities to expand and update their practice in order to keep up with the new, emerging practices. However, there is a tension between the perceived need to take time out of work for professional development and to ensure adequate human resource is available to carry on business as usual with the laboratory. To reduce this tension, technologyenhanced learning should be designed in ways that fits around and aligns with work tasks. Through effective design thinking and creative use of technology, learning experiences can be designed and implemented in ways that make learning almost invisible in professional roles.

The second tension focuses on the problems with implementing new ways of working with limited opportunity to re-configure the work environment to support new practice. We provide evidence that professional learning is ultimately structured by the workplace. For learning to be effective, people have to adapt the resources, processes and practices that make up the workplace itself. This means that capacity building has to focus on two broad and connected areas: first knowledge and skills development and second adapting the workplace. These findings have important implications for the design of Technology-enhanced Learning. Learning skills and knowledge in itself is not sufficient to tackle AMR. For professional learning to be effective, skills training has to be accompanied by a reflection on and re-organization of the work environment. This could be achieved by designing professional learning events in ways that allow policymakers, senior laboratory professionals and lab professionals to reflect upon and reimagine how work is structured and how new practices (e.g., data use and interpretation) can be built and sustained. This idea of reflecting on work tasks and restructuring of workplace aligns with Williams' (2014, p. 147) notion of "becoming" a practitioner, where professionals shape the systemic practices and ways of doing things associated with expertise. This finding is also in agreement with Fuller and Unwin's conclusion that both organizational and pedagogical features of professional learning characterize the nature and quality of the learning experience (Fuller and Unwin, 2003; Unwin and Fuller, 2004).

The third tension concerned the need for professionals to work in an inter-related way, as new work practices evolve and rigid professionals hierarchies alongside long-established work norms and processes. However, this way of working is different from conventional forms of practice, An important aspect of adapting the workforce is to consider first how work is performed as a distributed activity and how people inter-relate at a distance and second the diverse professional expertise needed and how people work across these areas of professional expertise. Lack of co-ordinated joint action around a key object-in this case AMR Data-hinders effective working and learning. Different groups with diverse job roles within the system work in silos and organizations have to develop systematic ways to work and learn collaboratively. Each professional needs to understand how his or her work fits within the system and how it inter-relates to the work of other professionals to ensure people have shared values. Technology-enhanced Learning can be designed in ways that use technical platforms to connect dispersed professionals.
These platforms could be implemented in ways that encourage each professional to consider their position in the overall system and how they can better inter-relate with others. By focusing on the position of each individual in the whole system, professionals could identify new roles that need to be created to allow them to bridge across sites. Interdependence of these new roles can be fostered across professional groups, improving collaboration and cooperation.

In conclusion, this study highlights the importance of the professionals' "voice" as a lens through which researchers document their reality. While quasi-ethnographic methods are not new, this methodology is not widely adopted in technologyenhanced learning research, where design is emphasized. Ethnographic data not only allows TEL researchers to maintain criticality in ways in which TEL and learning can add value in their professional lives-while closely attending to the voices of participants and their experiences. There is an urgent need to shift the orientation of research in TEL, moving focus from the design of learning activity and technology tools toward examining work practice in the words of the practitioners and mapping supporting technologies.

In line with Littlejohn and Pammer-Schindler (forthcoming), our work calls for a fundamental shift in the orientation of research in Technology Enhanced Learning, moving attention toward work practice and mapping supporting technologies around this, rather than focusing primarily on the technology and planning learning activity with technology tools.

There are a number of limitations of this study. First, it is acknowledged that the interviews were conducted in English, which is not the participants' first language. Second, the workplaces visited are live healthcare sites, therefore access had to be limited as work priorities arose. Third the study reflects the views of a number of professionals in specific contexts, therefore any generalization to wider professional communities should take this limitation into consideration. As the study of Cox et al. (2017) suggests, educational interventions around AMR are feasible in LMICs, but they need to be contextualized in the specific context.

\section{DATA AVAILABILITY STATEMENT}

The datasets for this study will not be made publicly available because the qualitative data used in the analysis for this article is embargoed to protect the anonymity of the subjects, as per the ethical consent signed by those we interviewed.

\section{ETHICS STATEMENT}

This study was carried out in accordance with the recommendations of ethics research guidelines, HREC Committee, Open University, with written informed consent from all human subjects prior to interview or photography. Ethical approval was granted from the Open University HREC Committee, which is the ethical approval committee for education research at the Open University, UK. An application outlining the research method and all instruments as well 
as the data gathering, storage and usage procedure was approved in 2018. All subjects gave written informed consent in accordance with the Declaration of Helsinki. The qualitative data is held by the Open University, UK in accordance with these guidelines.

\section{AUTHOR CONTRIBUTIONS}

$\mathrm{AL}$ was the principal investigator, providing conceptual oversight of the study, leading the research design, gathering, and analyzing the data. KC was the lead researcher, providing research oversight, gathering, and analyzing the data. HK was the researcher, supporting the data analysis, and interpretation.

\section{REFERENCES}

Boshuizen, H. P. (2004). "Does practice make perfect?" in Professional Learning: Gaps and Transitions on the Way from Novice to Expert (Dordrecht: Springer), 73-95. doi: 10.1007/1-4020-2094-5_5

Boud, D., and Garrick, J. (eds.). (1999). 'Understandings of workplace learning," in Understanding Learning at Work (London: Routledge), 29-44.

Charitonos, K., Littlejohn, A., Seal, T., and Kaatrakoski, H. (2018). Ways of Working and Learning in AMR Surveillance Systems in LMICs: Findings from the Scoping Phase. The Open University.

Colley, H., Hodkinson, P., and Malcolm, J. (2002). Non-Formal Learning: Mapping the Conceptual Terrain, a Consultation Report. Leeds: University of Leeds, 5.

Cox, J. A., Vlieghe, E., Mendelson, M., Wertheim, H., Ndegwa, L., Villegas, M. V., et al. (2017). Antibiotic stewardship in low- and middle-income countries: the same but different. Clin. Microb. Infect. 23, 812-818. doi: 10.1016/j.cmi.2017.07.010

Davison, H. C., Woolhouse, M. E., and Low, J. C. (2000). What is antibiotic resistance and how can we measure it? Trends Microbiol. 8, 554-559. doi: 10.1016/S0966-842X(00)01873-4

Engeström, Y. (2007). "From communities of practice to mycorrhizae," in Communities of Practice: Critical Perspectives, eds J. Hughes, N. Jewson, and L. Unwin (London: Routledge).

Engeström, Y. (2015). Learning by Expanding: An activity-Theoretical Approach to Developmental Research, 2nd Edn. Cambridge: Cambridge University Press. doi: $10.1017 /$ СBO9781139814744

Eraut, M. (2011). Informal learning in the workplace: evidence on the real value of work-based learning. Develop. Learn. Org. 25, 8-12. doi: $10.1108 / 14777281111159375$

Fuller, A., and Unwin, L. (2003). Learning as apprentices in the contemporary UK workplace: creating and managing expansive and restrictive participation. $J$. Educ. Work 16, 407-426. doi: 10.1080/1363908032000093012

Hadwin, A. F., Järvelä, S., and Miller, M. (2011). "Self-regulated, co-regulated, and socially shared regulation of learning," in Educational Psychology Handbook Series. Handbook of Self-Regulation of Learning and Performance, Vol. 30, eds B. J. Zimmerman and D. H. Schunk (New York, NY: Routledge/Taylor \& Francis Group), 65-84.

Hager, P. (2004). Lifelong learning in the workplace? Challenges and issues. J. Workplace Learn. 16, 22-32. doi: 10.1108/13665620410521486

Hall, W. (2018). Superbugs: An Arms Race against Bacteria. Harvard University Press. doi: $10.2307 /$ j.ctv2867t5

Illeris, K. (2011). "Workplaces and learning," in The SAGE Handbook of Workplace Learning, eds M. Malloch, L. Cairns, K. Evans, and B. N. O'Connor (London: SAGE), 32-45. doi: 10.4135/9781446200940.n3

Littlejohn, A., Hood, N., Milligan, C., and Mustain, P. (2016a). Learning in MOOCs: motivations and self-regulated learning in MOOCs. Internet Higher Educ. 29, 40-48. doi: 10.1016/j.iheduc.2015.12.003

Littlejohn, A., and Margaryan, A. (eds.) (2014). "Technology-enhanced professional learning," in International Handbook of Research in Professional and Practice-Based Learning (Dordrecht: Springer), 1187-1212. doi: 10.1007/978-94-017-8902-8_43

\section{FUNDING}

This research was funded by UK Aid through Department of Health and Social Care, Fleming Fund 2018-2019.

\section{ACKNOWLEDGMENTS}

The authors wish to thank all the participants of this study. Thanks also to Mott Macdonald and the UK Government Department for Health and Social Care Fleming Fund for funding this study and the support they provided during the country visit. We are also grateful to Mr. Tim Seal, Senior Project Manager, for his work on project management and in-country data collection.

Littlejohn, A., Milligan, C., Fontana, R. P., and Margaryan, A. (2016b). Professional learning through everyday work: how finance professionals self-regulate their learning. Vocations Learn. 9, 207-226. doi: 10.1007/s12186-015-9144-1

Littlejohn and Pammer-Schindler (forthcoming). Handbook of Research Approaches on Workplace Learning, eds D. Gijbels and C. Hartelis (Springer).

Morency-Potvin, P., Schwartz, D. N., and Weinstein, R. A. (2017). Antimicrobial stewardship: how the microbiology laboratory can right the ship. Clin. Microbiol. Rev. 30, 381-407. doi: 10.1128/CMR.00066-16

O'Neil, J. (2014). “The review on antimicrobial resistance," in Antimicrobial Resistance: Tackling a Crisis for the Health and Wealth of Nations. UK, HM Government Report: AMR. ORG, 1-20.

Paavola, S., and Hakkarainen, K. (2005). The knowledge creation metaphorAn emergent epistemological approach to learning. Sci. Educ. 14, 535-557. doi: 10.1007/s11191-004-5157-0

Paavola, S., Lipponen, L., and Hakkarainen, K. (2004). Modeling innovative knowledge communities: a knowledge-creation approach to learning. Rev. Educ. Res. 74, 557-576. doi: 10.3102/00346543074004557

Sayed, S., Cherniak, W., Lawler, M., Tan, S. Y., El Sadr, W., Wolf, N. et al. (2018). Improving pathology and laboratory medicine in low-income and middle-income countries: roadmap to solutions. Lancet 391, 1939-1952. doi: 10.1016/S0140-6736(18)30459-8

Tuomi, J., and Sarajärvi, A. (2009). Laadullinen tutkimus ja sisällönanalyysi [Qualitative Research and Content Analysis], 6th Edn. Helsinki: Tammi.

Tynjälä, P. (2008). Perspectives into learning at the workplace. Educ. Res. Rev. 3, 130-154. doi: 10.1016/j.edurev.2007. 12.001

Unwin, L., and Fuller, A. (2004). "Expansive learning environments: integrating organizational and personal development," in Workplace Learning in Context (Routledge), 142-160.

Williams, J. (2014). Teacher educator professional learning in the third space: Implications for identity and practice. J. Teach. Educ. 65, 315-326. doi: $10.1177 / 0022487114533128$

Wilson, M. L., Fleming, K. A., Kuti, M. A., Looi, L. M., Lago, N., and Ru, K. (2018). Access to pathology and laboratory medicine services: a crucial gap. Lancet 391, 1927-1938. doi: 10.1016/S0140-6736(18)30458-6

World Health Organization (2019). Antimicrobial Resistance. Available online at: https://www.who.int/antimicrobial-resistance/en/ (accessed August $1,2019)$.

Conflict of Interest: The authors declare that the research was conducted in the absence of any commercial or financial relationships that could be construed as a potential conflict of interest.

Copyright (c) 2019 Littlejohn, Charitonos and Kaatrakoski. This is an open-access article distributed under the terms of the Creative Commons Attribution License (CC $B Y)$. The use, distribution or reproduction in other forums is permitted, provided the original author(s) and the copyright owner(s) are credited and that the original publication in this journal is cited, in accordance with accepted academic practice. No use, distribution or reproduction is permitted which does not comply with these terms. 